\title{
ArcheoSciences
}

Revue d'archéométrie

$30 \mid 2006$

Varia

\section{Mortiers de chaux dolomitique avec adjonction de kaolin cuit : l'expérience génoise}

Dolomitic lime mortars with addition of burned kaolin: the genoese experience

\section{Tiziano Mannoni, Gianluca Pesce et Rita Vecchiattini}

\section{(2) OpenEdition}

\section{Édition électronique}

URL : http://journals.openedition.org/archeosciences/165

DOI : 10.4000/archeosciences. 165

ISBN : 978-2-7535-1595-6

ISSN : 2104-3728

Éditeur

Presses universitaires de Rennes

Édition imprimée

Date de publication : 31 décembre 2006

Pagination : 67-79

ISBN : 978-2-7535-0456-1

ISSN : 1960-1360

\section{Référence électronique}

Tiziano Mannoni, Gianluca Pesce et Rita Vecchiattini, « Mortiers de chaux dolomitique avec adjonction de kaolin cuit : l'expérience génoise », ArcheoSciences [En ligne], 30 | 2006, document 6, mis en ligne le 31 décembre 2008, consulté le 30 avril 2019. URL : http://journals.openedition.org/

archeosciences/165; DOI : 10.4000/archeosciences.165 


\title{
Mortiers de chaux dolomitique avec adjonction de kaolin cuit : l'expérience génoise
}

\author{
Tiziano Mannoni*, Gianluca Pesce** et Rita Vecchiattini***
}

\begin{abstract}
Résumé : La recherche sur les matériaux utilisés à Gênes, de la fin du Moyen Âge aux premières décennies du xx siècle, dans la réalisation des mortiers ou de revêtement d'œuvres murales, naît de surprenants résultats de certaines enquêtes archéologiques conduites par des membres de l'Institut d'Histoire de la Culture Matérielle (ISCuM) de cette même ville ligure, à partir de la deuxième moitié des années 1980. En effet, ces recherches ont mis en évidence comment l'utilisation intense de la chaux dolomitique et l'adjonction - si nécessaire - de kaolin cuit en qualité de liant hydraulique ont donné naissance à la construction d'une bonne partie de la ville portuaire avec des résultats parfois surprenants pour la qualité des produits finaux qui ont été obtenus. Le cas du port de Gênes, construit à la fin du Moyen Âge, est, dans ce sens, emblématique car les mortiers, après environ cinq siècles de leur réalisation et de permanence dans un environnement agressif, démontrent encore aujourd'hui faire preuve d'une mécanique de résistance élevée aux agents corrosifs chimiques et biologiques.

Cette contribution entend mettre en évidence le niveau de la recherche menée sur ces matériaux par les chercheurs de l'ISCuM et de l'Université de Gênes; recherche qui est toujours en cours et qui se poursuit dans une direction fortement multidisciplinaire, selon un processus cognitif qui a conduit des chantiers archéologiques aux laboratoires de minéralogie et de pétrographie de la Faculté de Sciences naturelles, en passant par les laboratoires d'ingénierie des matériaux de l'Université de Gênes.
\end{abstract}

Abstract: The research about the materials used at Genoa from middle age to Nineteen century for the production of mortars and plasters, beginning from the interesting results of some archaeological researches conduct from the Institute of History of the Material's Culture (I.S.Cu.M.), in the firs half of Eighty years of last century.

This researches have point out the large use of the dolomitic lime and the use of burned kaolin like pozzolanic material in the building construction, and have highlighted the surprising results of some dough produced during the city construction. An example of this results are the mortars of medieval harbour that still now give, after five century of the permanence in an aggressive environment, an high mechanical resistance and an high resistance to the chemical and biological attack.

With this papers we want underline the results of this researches that have been conduct in a multidisciplinary method from the archaeological yard, through the mineralogical laboratories, to the laboratories of materials engineering of the University of Genoa.

Mots clés : Chaux, kaolin, mortiers, $\mathrm{XRD}, \mathrm{CO}_{2}$, vapeur d'eau, ettringite.

Key words : Lime, kaolin, mortars, $X R D, C O_{2}$, water vapor, ettringite.

Ce papier a pour but de mettre en évidence l'état de la recherche menée à Gênes par plusieurs chercheurs de l'Université et de l'Institut d'Histoire de la Culture Matérielle (ISCuM), sur les matériaux utilisés - dans cette même ville ligure - de la fin du Moyen Âge aux premières décennies $\mathrm{du} \mathrm{xx}^{\mathrm{e}}$ siècle, pour la réalisation des mortiers utilisés dans l'édification de la ville.

* Institut d'Histoire de la Culture Matérielle, via di Sottoripa, 5/1, 16124 Gênes (Italie). (mannoni@iscum.it)

** Institut d'Histoire de la Culture Matérielle, via di Sottoripa, 5/1, 16124 Gênes (Italie). (gianluca.pesce@libero.it)

*** Département de Science pour l'Architecture - Faculté d'Architecture (Université de Gênes), Stradone S. Agostino, 37, 16123 Gênes (Italie). (rita. vecchiattini@libero.it) 
L'étude est née des résultats surprenants de certaines enquêtes archéologiques menées par les mêmes membres de l'ISCuM à partir du 1985 (Mannoni, 1988 et Vecchiattini, 1998) qui ont mis en évidence l'utilisation intense de la chaux dolomitique et l'adjonction - si nécessaire - de kaolin cuit en qualité de liant hydraulique qui ont donné naissance à la construction d'une bonne partie de la ville portuaire avec des résultats parfois surprenants pour la qualité des produits finaux qui ont été obtenus. Le cas du port de Gênes, construit à la fin du Moyen Âge, est, dans ce sens, emblématique car les mortiers, à environ cinq siècles de leur réalisation et en permanence dans un environnement agressif, démontrent encore aujourd'hui leur résistance élevée aux agents corrosifs chimiques et biologiques (Mannoni, 1988, 138-139).

Comme nous avons déjà soulignée, les recherches sur l'utilisation de ces deux matériaux qui, à l'origine, sont nées dans le milieu archéologique, se sont, au cours des années, progressivement déplacées vers les laboratoires de minéralogie et pétrographie et plus récemment vers les laboratoires d'Ingénierie des Matériaux selon un processus d'étude multidisciplinaire qui a donné des résultats brièvement exposés ici.

Dans la suite de cet article, on présentera, en effet, les résultats atteints aujourd'hui par la recherche la plus avancée en matière de pratique d'utilisation de la chaux dolomitique (première partie de l'exposé) et du kaolin cuit (deuxième partie); recherche qui est toujours en cours et que l'on espère pouvoir approfondir et documenter avec force détails dans l'avenir.

L'exposition de la recherche sur les chaux dolomitiques démarre notamment par une lecture géologique du territoire intéressé et par la description de l'étude des sources des matériaux que l'on peut trouver aujourd'hui à la proximité immédiate de la ville de Gênes, pour arriver finalement aux résultats des expériences de laboratoire menées à la Faculté d'Ingénierie où ont été explorées les conditions de cuisson des calcaires dolomitiques utilisés pour la production de ces liants. Les études sur le kaolin cuit présentent, en revanche, des réflexions sur les produits finaux et sur les modalités d'utilisation de ce matériau, qu'il est possible de faire à partir d'une large base de données constituée par les résultats des diffractométries des rayons $\mathrm{X}$ réalisées sur un nombre élevé de mortiers produits à Gênes à l'époque préindustrielle, et échantillonnées au cours des différentes activités des chercheurs.

\section{LA CHAUX DOLOMITIQUE}

La ville de Gênes (Italie) enclavée entre les monts et la mer, se trouve à quelques kilomètres d'une importante chaîne de montagnes qui se développe perpendiculairement à la mer définissant ainsi deux vallées : la Vallée Polcevera du côté de Gênes et la vallée Varenna à l'Ouest. Il s'agit de celle qui est définie comment la Zona Sestri Voltaggio (Fig. 1) puisque, de l'agglomération de Sestri Ponente, désormais partie intégrante de la ville de Gênes, elle se développe sur une cinquantaine de kilomètres vers les pays de l'intérieur jusqu'au village de Voltaggio. Cette zone, autrefois considérée comme la frontière géologique entre les Alpes à l'Ouest et les Apennins à l'Est, est constituée de trois différentes entités : l'Unité du Mont Gazzo-Isoverde, l'Unité du Mont Figogna et l'Unité de Cravasco-Voltaggio (Società Geologica Italiana 1994). La première Unité qu'on rencontre en s'éloignant de la mer et de Gênes est celle du Mont Gazzo-Isoverde, constituée essentiellement de roche dolomitique déposée en couches d'épaisseur métrique entrecoupées de niveaux marneux. Le mont, compté parmi les plus anciens de la Ligurie (trias et jurassique, 250-140 millions d'années; Allasinaz et al. 1971), est constitué de dolomie de couleur grise avec des traces de carbonate de magnésium concentré en veines de formation marine. Il s'agit d'une formation rocheuse d'origine sédimentaire dont la constitution remonte à deux centaines de millions d'années, dans un environnement marin complètement différent de l'actuel, caractérisé par une faible profondeur, une variation sensible des marées et des températures qui aujourd'hui seraient qualifiées de tropicales.

Il est certain que la proximité de la formation rocheuse et de l'agglomération de Gênes ainsi que la possibilité d'utiliser un moyen confortable de transport par voies d'eau, le long du torrent dans la première phase du parcours puis par mer, a contribué voire déterminé une large utilisation de la roche dolomitique comme matière première pour la production de la chaux dans la région de Gênes. Des analyses de laboratoire ont en fait démontré que dès le $\mathrm{xI}^{\mathrm{e}}$ siècle on utilisait à Gênes la chaux dolomitique ${ }^{1}$, comme liant dans des mortiers, et le premier document écrit (Archives d'État de Gênes, Notai Ignoti, enveloppe 1/XVI, attribué à Pietro Ruffo, c. 19r), connu actuallement, parlant de la présence d'un four à chaux au flanc du mont Gazzo, remonte à 1214 . Dès lors, mais peut-être même antérieurement, l'utilisation génoise de la chaux dolomitique a été pratiquée diffusément jusqu'à la deuxième moitié du XIx ${ }^{e}$ siècle, quand l'installation de la voie ferrée de liaison avec la plaine du Pô a favorisé la diffusion d'un nouveau matériau liant qui, au cours de ces années, était en train de s'affirmer en Europe et en Italie : le ciment. À la suite de cela, la production artisanale a été confrontée à de nouvelles demandes du marché et, vers le

1. Par chaux dolomitique on entend celle qui contient une teneur en Oxyde de Magnésium $\mathrm{MgO}>30 \%$ et donc une quantité d'Oxyde de Calcium $\mathrm{CaO}<70 \%$ (Norme technique C.N.R. n. 129 du 30 septembre 1988 Caratteristiche tecniche e requisiti delle calci aeree). 


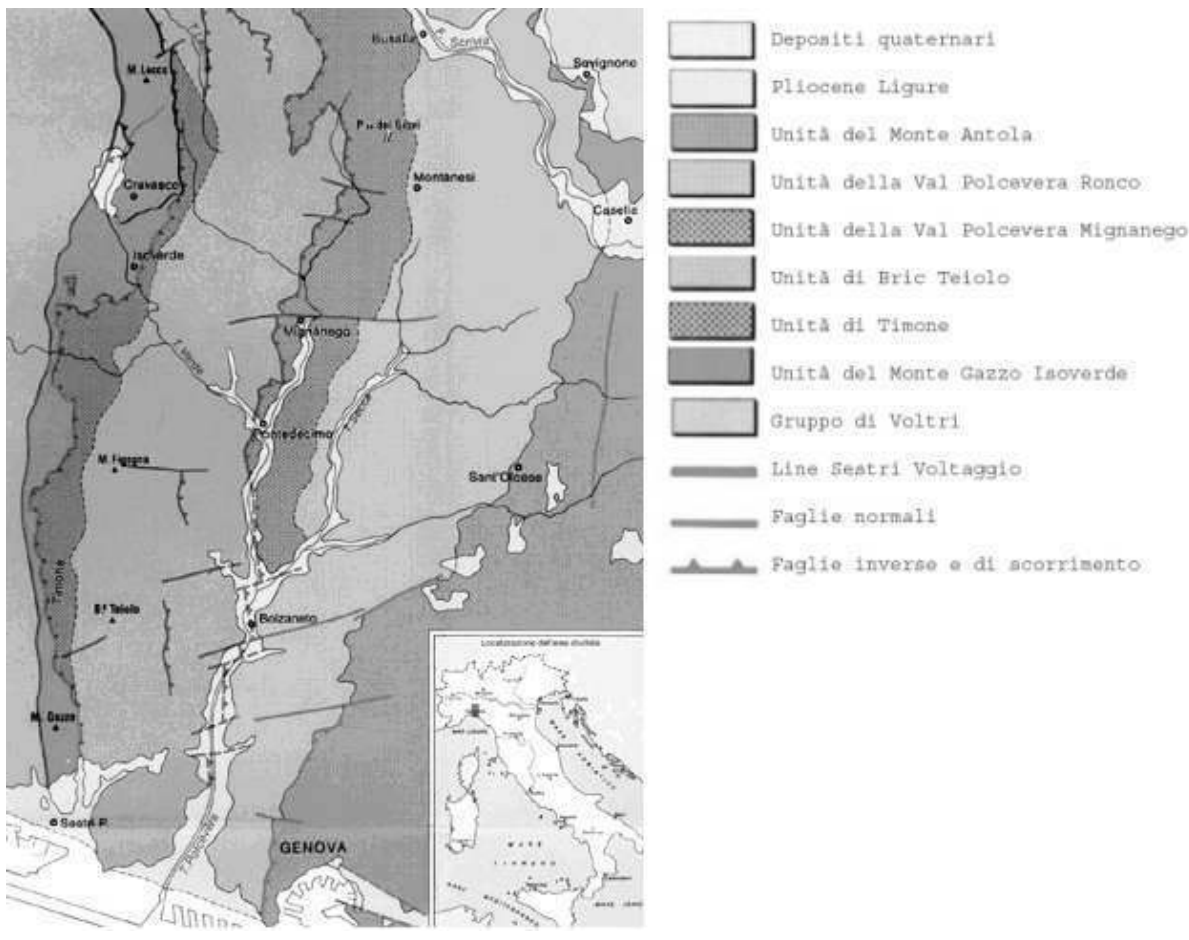

Figure 1 : Schéma des unités tectoniques de la zone de Sestri Ponente - Gênes (extrait de M. Marini 1997, échelle 1/100 000).

Figure 1: Tectonic units of Sestri Ponente area (from Marini 1997, scale 1/100000)

début du $\mathrm{Xx}^{\mathrm{e}}$ siècle, l'imposante production de chaux dolomitique au mont Gazzo céda la place à la production de blocaille pour la construction des routes.

Ce fut la période au cours de laquelle, aussi bien en Italie que dans d'autres pays européens, allait commencer une conversion progressive des petits fours locaux qui, souvent, furent remplacés ou transformés selon les besoins en une production à caractère industriel (Tamagno, 1987). Toutefois, pendant que dans d'autres zones de la Ligurie on assiste encore aujourd'hui à un tel processus d'adaptation des établissements et à une progressive transformation de la production, il semble que l'activité s'est arrêtée au mont Gazzo au début du $\mathrm{xx}^{\mathrm{e}}$ siècle en laissant une zone préindustrielle presque intacte (Fig. 2) où se reconnaissent encore les anciennes carrières, les fours à chaux, les maisons des propriétaires ou des locataires des fours eux-mêmes, les abris des ouvriers chargés de suivre la cuisson des matériaux. Ce sont précisément ces ouvrages, encore conservés dans leurs formes et organisation originales, qui ont attiré l'attention des spécialistes en histoire de la production de la chaux à Gênes et ont introduit indirectement à l'étude de la chaux dolomitique actuellement en cours à l'Université de Gênes depuis 1992, mais non de manière continue. Il est tout de suite apparu clairement que l'étude de ces ouvrages de production devait être nécessairement multidisciplinaire et se servir non seulement des apports de l'histoire de la production, de l'archéologie, de l'architecture, de l'exégèse des sources, de la technologie constructive, mais aussi de la physique, de la géologie, de la chimie, de la science et de l'ingénierie des matériaux. C'est seulement à partir d'une vision parallèle et contemporaine des diverses méthodes que nous commençons à comprendre aujourd'hui le phénomène lié à la fortune et à la ruine de la chaux dolomitique, non seulement à Gênes mais en général en Italie (surtout dans la région du Lago Maggiore et des Dolomiti; Commissione Europea e Centro Comune di Ricerca 1995) et en Europe (en particulier la production et l'utilisation de chaux dolomitique sont documentées en Espagne, France, Allemagne et Angleterre, surtout entre les XIV ${ }^{e}$ et XVII ${ }^{e}$ siècles : Newton, 1987; Manzano et al. 2000).

Les recherches sur les sources écrites ont fait ressortir une réalité jusqu’à hier ignorée : jusqu’à la seconde moitié du $\mathrm{XIX}^{\mathrm{e}}$ siècle, on jugeait utile, et souvent même nécessaire, la présence d'une quantité optimale de magnésium dans les mélanges des mortiers. En revanche, à partir des premières années du siècle dernier, l'utilisation des liants magnésiens et dolomitiques a été totalement abandonnée car ces derniers étaient considérés comme des produits de qualité inférieure. 


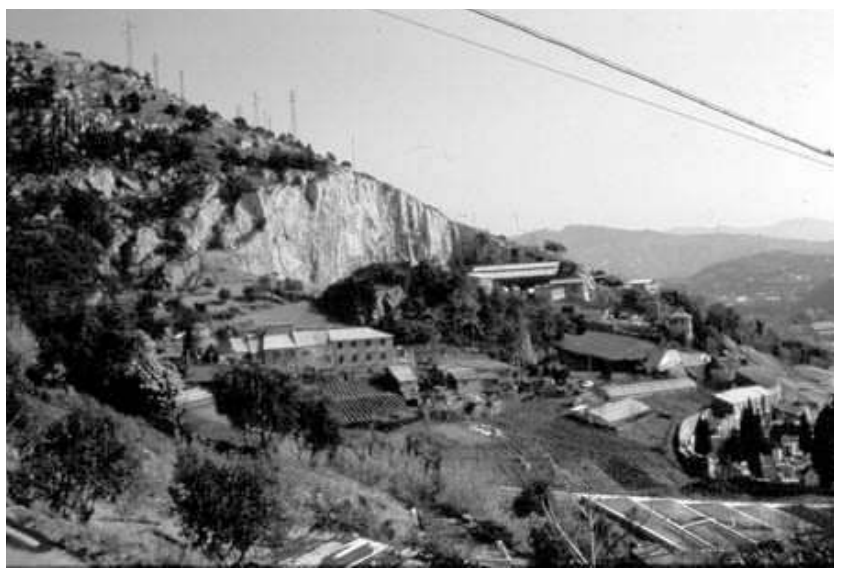

Figure 2: Fours à chaux dans la localité Vecchie Fornaci à Sestri Ponente, Gênes.

Figure 2: Lime kilns of Vecchie Fornaci, Sestri Ponente

Les sources sont nombreuses et en grande partie crédibles, il est donc possible d'admettre l'existence d'un changement réel et radical de la qualité du produit. Cependant, il est surprenant d'évaluer la durée de bon état de conservation de la plupart des mortiers génois réalisés avec la chaux dolomitique et, bien que s'agissant d'un jugement de type qualitatif et non quantitatif, il faut se souvenir qu'il est basé sur la preuve du temps et qu'il est sûrement en mesure de donner des réponses dignes de foi, du moins pour ce qui est de la durée des matériaux de construction. C'est pour comprendre les raisons de l'abandon de tels matériaux qu'ont été conduites récemment dans la recherche deux nouvelles disciplines : la science et l'ingénierie des matériaux qui opèrent de façon complètement différente des thèmes archéométriques plus traditionnels. Dans cette nouvelle optique, l'étude de la chaux dolomitique est fondée sur la reproduction, en laboratoire, de processus de production, en cherchant à évaluer l'influence des divers facteurs concernés (matière première, température, atmosphère gazeuse...) sur les caractéristiques finales des produits.

De l'analyse historico-archéologique conduite sur le territoire ligure il est ressorti que le présumé changement de qualité des produits a eu lieu précisément en concomitance avec la révolution de la technologie de production précédemment rappelée. Des fours intermittents ${ }^{2}$ de type préindustriel comme ceux qui sont encore présents au mont

2. Les fours sont dits intermittents quand, pour leur bon fonctionnement, des phases distinctes et successives sont nécessaires : chargement, cuisson, refroidissement et déchargement. L'installation type qui caractérise les unités de production intermittentes, alimentées au bois, est constituée par une structure cylindrique verticale, se terminant par une pseudo-voûte et surmontée par une cheminée. (Vecchiattini, 1998).
Gazzo, on est passé aux fours continus ${ }^{3}$ de type industriel. Cette progressive substitution technologique commune à bien des zones de production, en Italie et à l'étranger, ne s'est jamais produite au mont Gazzo, même si ne manquèrent pas les tentatives d'entrepreneurs téméraires, qui ont par ailleurs toujours échoué, à implanter des fourneaux dotés de systèmes innovants. Les modalités de production de chaux dolomitique au mont Gazzo furent toujours les mêmes pendant des siècles jusqu'à ce qu'elles deviennent vraiment trop peu compétitives sur le marché national. Le dernier démontage d'un four à chaux au mont Gazzo remonte en 1914.

Le fait d'avoir associé le changement de la qualité des produits à la variation des typologies de fours à chaux a permis de faire une avancée importante dans le domaine de l'étude et de mener une enquête, entre autres éléments, sur le combustible employé durant différentes périodes et pour les différentes typologies. Les données étaient connues dès le début de la recherche documentaire et il n'est pas difficile de prouver que les fours du type préindustriel étaient alimentés avec des fagots de petit bois; on utilisait en particulier des essences comme le hêtre, le pin sauvage, l'aune, le genévrier et le rouvre, tandis que les fours continus furent d'abord alimentés à la houille et seulement plus tard aux huiles minérales et gaz naturels. L'influence du type de combustible utilisé sur le produit oxydé de la décomposition de la dolomie est déjà signalée, en marge d'autres indications, par divers auteurs qui toutefois l'associent surtout aux différents pouvoirs calorifiques du combustible, sans analyser le thème du point de vue scientifique (Ghersi, 1903; Vicat, 1856; Maede, 1909).

\section{LA RECHERCHE DE LABORATOIRE SUR LA CHAUX DOLOMITIQUE}

Dans le domaine des études de l'ingénierie des matériaux, il est intéressant d'approfondir l'analyse de l'influence, tant de la température que du type de combustible, sur le produit de la décomposition, à partir de l'étude des atmosphères gazeuses, présentes à l'intérieur des fours alimentés avec divers combustibles, effectuée sur la base de calculs classiques de la chimie de la combustion ${ }^{4}$.

3. Les fours sont définis continus quand les quatre phases de production (chargement, cuisson, refroidissement, déchargement) ont lieu simultanément sans devoir éteindre et rallumer le four. Il s'agit, pour la plupart, de fours rectangulaires ayant une capacité fusiforme dans lequel le chargement de matière première et de combustible est prévu par le haut et la cuisson du matériel se produit environ dans la partie moyenne du four tandis que le déchargement se fait par le bas à travers une grille de fer. (Lenormand et al., 1839, p. 512; Pareto et al., 1885, pages 61-62).

4. Analyse développe dans le cadre du Doctorat de Recherche en Ingénierie des Matériaux, XV cycle du Polytechnique de Milan tenu par 
Létude des atmosphères gazeuses a permis de déterminer des différences non négligeables dans les apports des différentes composantes des fumées (Tableau 1).

La différence entre la quantité d'anhydride carbonique et la quantité de vapeur d'eau apparaît clairement, déjà à partir de la composition des fumées théoriques : pendant la combustion le bois produit moins d'anhydride carbonique mais plus de vapeur d'eau par rapport la houille.

Toutefois, la donnée la plus significative ressort du calcul des pressions partielles, liées à la quantité totale des fumées théoriques (par le bois $5.378 \mathrm{Nm} 3 / \mathrm{kg}$ ), on en déduit que la pression partielle de la vapeur d'eau dans un four alimenté au bois est presque le double de celle présente dans un four alimenté à la houille, tandis que les valeurs de la pression partielle de l'anhydride carbonique sont tout à fait comparables dans les deux cas (Tableau 2).

Laissant de côté les pressions partielles d'anhydride sulfurique et d'azote, nettement basses et donc n'ayant aucune influence en comparaison de la masse de la dolomie, on a successivement analysé l'effet de l'anhydride carbonique et de la vapeur d'eau sur la microstructure des grains d'oxyde de calcium et d'oxyde de magnésium produits durant la décomposition de la dolomie. Pour ce faire, il a fallu simuler en laboratoire la cuisson de la pierre à chaux dans deux différentes conditions de milieu gazeux et, successivement, analyser les produits oxydés pour en évaluer les similitudes et/ou les différences.

La matière première, à savoir la pierre à chaux, a été prélevée dans une carrière historique abandonnée depuis longtemps mais encore visible sur le flanc sud du mont Gazzo. Le prélèvement des échantillons de la roche a été réalisé en trois points différents au fond de la carrière, en ayant bien soin d'éliminer préalablement la partie superficielle de la roche, la plus exposée à l'altération atmosphérique.

La cuisson de la pierre dans le four a été reproduite en laboratoire à l'aide d'une balance pour l'analyse thermique
TG-DSC, Netzsch Gerätebau avec un système d'acquisition STA 409, le four de laquelle on a envoyé un flux constant de seul $\mathrm{CO}_{2}$, dans le cas d'une atmosphère liée au combustible de houille, et un flux de $\mathrm{CO}_{2+} \mathrm{H}_{2} \mathrm{O}$ en phase gaz, dans le cas d'une atmosphère liée au combustible de bois.

Dans la littérature scientifique, on connaissait déjà l'effet de l'anhydride carbonique sur la microstructure de l'oxyde de calcium, produit par la décomposition du carbonate de calcium (Bamford et al., 1980). En général, nous pouvons résumer en disant que la microstructure et la nature des produits solides sont étroitement dépendantes de la pression partielle de $\mathrm{CO}_{2}$ proche de l'interface de réaction et de la température de décomposition, en particulier à basse pression d'anhydride carbonique, l'oxyde a encore la forme cristalline initiale, tandis que les hautes pressions d'anhydride carbonique favorisent le frittage des grains formant des grains de plus grandes dimensions et de forme différente. En revanche, l'effet de la vapeur d'eau sur la microstructure de l'oxyde de magnésium n'était pas connu, c'est pourquoi on a exploré avec une attention particulière la première phase de la décomposition pendant laquelle se produit la dissociation de $\mathrm{CaMg}\left(\mathrm{CO}_{3}\right)_{2}$ avec formation de $\mathrm{MgO}$ et la réorganisation des cristaux en $\mathrm{CaCO}_{3}$. À la formule $P_{p \mathrm{CO} 2}$ de 1 atm, l'intervalle de température au moment où se produit la première partie de la décomposition est compris entre 913 et $973 \mathrm{~K}$, c'est la raison pour laquelle on a choisi sept valeurs de température, tous les $10 \mathrm{~K}$, de 913 à $973 \mathrm{~K}$, auxquelles on a effectué autant de décompositions isothermes, toutes dans les mêmes conditions expérimentales, afin de confronter les produits oxydés finaux.

Les échantillons de matières premières, consistant en 55 $\pm 2 \mathrm{mg}$ de poudre de dolomie, ont été décomposés dans des creusets ${ }^{5}$ de Knudsen (Margrave, 1960; Mohazzabi et al., 1974) à l'intérieur de la même balance pour l'analyse thermogravimétrique. Après avoir positionné deux creusets, complètement remplis, l'un de poudre de dolomie et l'autre

\begin{tabular}{|l|c|c|c|c|c|}
\cline { 2 - 6 } \multicolumn{1}{c|}{} & $\begin{array}{c}\mathrm{CO}_{2} \\
{\left[\mathrm{Nm}^{3} / \mathrm{kg}\right]}\end{array}$ & $\begin{array}{c}\mathrm{H}_{2} \mathrm{O} \\
{\left[\mathrm{Nm}^{3} / \mathrm{kg}\right]}\end{array}$ & $\begin{array}{c}\mathrm{SO}_{2} \\
{\left[\mathrm{Nm}^{3} / \mathrm{kg}\right]}\end{array}$ & $\begin{array}{c}\mathrm{N}_{2} \\
{\left[\mathrm{Nm}^{3} / \mathrm{kg}\right]}\end{array}$ & $\begin{array}{c}\mathrm{V}_{\mathrm{FT}} \text { totale } \\
{\left[\mathrm{Nm}^{3} / \mathrm{kg}\right]}\end{array}$ \\
\hline Bois & 0.926 & 0.675 & - & 0.001 & 5.378 \\
\hline Houille & 1.122 & 0.542 & 0.006 & 0.007 & 6.259 \\
\hline
\end{tabular}

Tableau 1 : Composition des gaz présents à l'intérieur des fours alimenté avec divers combustibles. En ce qui concerne la composition du bois, nous avons calculé une moyenne entre les essences qui, d'après les sources historiques, ont été utilisées.

Table 1: Gas composition in the kilns fed with different fuels.

Rita Vecchiattini auprès du Département de Construction, Urbanistique et Ingénierie des Matériaux de l’Université de Gênes.
5. Les creusets d'alumine et de forme cylindrique (diamètre interne d'environ $4 \mathrm{~mm}$ et hauteur d'environ $3 \mathrm{~mm}$ ) sont pourvus d'un couvercle plat d'aluminium avec un trou central de $0,1 \mathrm{~mm}$ de diamètre. 


\begin{tabular}{|l|c|c|c|c|}
\cline { 2 - 5 } \multicolumn{1}{c|}{} & $\begin{array}{c}\mathrm{P}_{\mathrm{CO} 2} \\
{[\mathrm{~mm} \mathrm{Hg}]}\end{array}$ & $\begin{array}{c}\mathrm{P}_{\mathrm{H} 2 \mathrm{O}} \\
{[\mathrm{mm} \mathrm{Hg}]}\end{array}$ & $\begin{array}{c}\mathrm{P}_{\mathrm{sO} 2} \\
{[\mathrm{~mm} \mathrm{Hg}]}\end{array}$ & $\begin{array}{c}\mathrm{P}_{\mathrm{N} 2} \\
{[\mathrm{~mm} \mathrm{Hg}]}\end{array}$ \\
\hline Bois & 130.805 & 95.381 & - & 0.113 \\
\hline Houille fossile & 136.183 & 65.752 & 0.696 & 0.836 \\
\hline
\end{tabular}

Tableau 2 : Pression partielle de $\mathrm{H}_{2} \mathrm{O}, \mathrm{CO}_{2}, \mathrm{SO}_{2}$ et $\mathrm{N}_{2}$ dans les différentes atmosphères gazeuses.

Table 2: Pressure of $\mathrm{H}_{2} \mathrm{O}, \mathrm{CO}_{2}, \mathrm{SO}_{2}, \mathrm{~N}_{2}$.

d'une égale quantité en poids de poudre de Kaolin calciné ${ }^{6}$, on a introduit dans le four un flux ${ }^{7}$ de $\mathrm{CO}_{2}[g]$ à température ambiante avec une rapidité constante de 35,10 \pm 0,05 $\mathrm{ml}$. $\min ^{-1}$.

Dans le cas de la simulation des fours alimentés au bois (Fig. 3) le flux de $\mathrm{CO}_{2}[g]$ humide a été obtenu en introduisant le $C O_{2}$, à la vitesse constante de 35,10 $\pm 0,05 \mathrm{ml} \cdot \mathrm{min}^{-1}$, dans de l'eau distillée, réchauffée à la température de $50^{\circ} \mathrm{C}$ pour éviter la condensation de $\mathrm{H}_{2} \mathrm{O}[\mathrm{g}]$, et laissant bouillonner le gaz pendant 30 minutes. Le pourcentage de $\mathrm{H}_{2} \mathrm{O}[\mathrm{g}]$ dans le flux de $\mathrm{CO}_{2}[g]$ a été calculé à température ambiante, égal à 4,0 $\pm 0,1 \%$ en volume et, pendant toute la durée des expériences, aucune déviation appréciable de telle valeur n’a été relevée. Enfin, à travers une canule, à la sortie du récipient de $\mathrm{H}_{2} \mathrm{O}[g]$, le $\mathrm{CO}_{2}[g]$ humide a été introduit dans le four de la thermobalance.

Dans les deux cas, dès que la pression de $\mathrm{CO}_{2}[g]$ dans le four a atteint la valeur de $1 \mathrm{~atm}$. (après 3 minutes environ), la phase de préchauffage des échantillons a commencé à $823 \mathrm{~K}$, température proche de celle du début de la décomposition, pendant $30 \mathrm{~min}$. sous le flux constant de $\mathrm{CO}_{2}[g]$. Dans la phase de réchauffement, la température a augmenté rapidement, à la vitesse de $50 \mathrm{~K} \cdot \mathrm{min}^{-1}$, jusqu'à atteindre les températures de décomposition isotherme précédemment déterminées, à savoir les températures comprises dans l'intervalle entre 913 et $973 \mathrm{~K}$. Toutes les décompositions isothermes ont été effectuées tant en flux de $\mathrm{CO}_{2}[g]$ qu'en flux de $\mathrm{CO}_{2}[g]+\mathrm{H}_{2} \mathrm{O}[g]$ pendant 32 heures. À leur terme, fixé pour des mesures de sécurité à au moins 3 heures après avoir atteint le plateau, on a introduit un flux d'azote à la vitesse de 52,91 $\pm 0,05 \mathrm{ml} \cdot \mathrm{min}^{-1}$, pour éliminer rapidement les autres gaz contenus dans le four et effectuer la phase de refroidissement des échantillons jusqu'à la température ambiante en atmosphère de gaz inerte, en évitant ainsi

6. On a choisi la poudre de kaolin calciné, comme matériau de référence, car sa température de décomposition est de $1350^{\circ} \mathrm{C}$ et par conséquent bien supérieure à celle de la dolomie (Stern et al., 1969). Il faut, en effet, que l'échantillon de référence, ne subisse aucune perte de poids dans les intervalles de température choisis pour les expériences en balance thermogravimétrique.

7. Le flux a été réglé par un fluximètre Aalborg-U.S.A. ayan conditions normales une portée d'air de $28,4 \mathrm{mlmin}^{-1}$. toute réaction ${ }^{8}$ qui pourrait compromettre les produits de la décomposition. Chaque test a été répété, dans les mêmes conditions 3 fois, et la reproductibilité des expériences a été évaluée à $98 \%$, sur la base de la déviation standard calculée toutes les 150 minutes de preuve.

La figure 4 montre les courbes d'intrusion du porosimètre à mercure concernant les produits solides finaux pour la première partie de la décomposition en $\mathrm{CO}_{2}[g]$ (tracé de couleur grise) et pour celle en $\mathrm{CO}_{2}[g]+\mathrm{H}_{2} \mathrm{O}[g]$ (tracé de couleur noire) à $913 \mathrm{~K}$. On a soustrait de la porosité les vides intergranulaires par conséquent les courbes ne montrent que les cavités à l'intérieur des particules décomposées. Comme on peut l'observer, la porosité totale des échantillons en milieu de $\mathrm{CO}_{2}[g]+\mathrm{H}_{2} \mathrm{O}[g]$, est plus importante $\left(0,164 \mathrm{~cm}^{3} \mathrm{~g}^{-1}\right)$ que celle des échantillons décomposés en atmosphère de seul $C_{2}[g]\left(0,150 \mathrm{~cm}^{3} \mathrm{~g}^{-1}\right)$. Elle est même plus élevée que la porosité théorique totale de $0,12 \mathrm{~cm}^{3} \mathrm{~g}^{-1}$. Cette donnée, associée à l'observation que la forme des grains obtenus par décomposition en atmosphère de $\mathrm{CO}_{2}[g]+\mathrm{H}_{2} \mathrm{O}[g]$ est presque égale à celles des particules de poudre de dolomie non décomposée, mène à la conclusion que les particules décomposées en milieu de $\mathrm{CO}_{2}[g]+\mathrm{H}_{2} \mathrm{O}[g]$ doivent être brisées. En accord avec cette hypothèse il y a la différente distribution des pores qui dans le cas des échantillons décomposés en milieu de $\mathrm{CO}_{2}[g]$ est unimodale avec un diamètre moyen des pores d'environ $0,05 \mu \mathrm{m}$, et dans le cas des échantillons décomposés en atmosphère de $\mathrm{CO}_{2}[g]+\mathrm{H}_{2} \mathrm{O}[g]$ est bimodale avec un diamètre moyen des pores d'environ $1 \mu \mathrm{m}$.

Des preuves de l'existence de vastes cavités à l'intérieur des grains de poussière décomposée en atmosphère de $\mathrm{CO}_{2}[g]+$ $\mathrm{H}_{2} \mathrm{O}[\mathrm{g}]$ ont été observées sur la surface externe des particules décomposées, même à basse température (fig. 5). Sur la figure, on peut observer une longue fissure large d'environ $0,2 \mu \mathrm{m}$ et longue de $8 \mu \mathrm{m}$. Les dimensions moyennes de ces défauts ne semblent pas changer de manière significative avec l'augmentation de la température de décomposition, mais leur nombre oui, comme on le déduit de la valeur de la porosité totale qui devient de $0,222 \mathrm{~cm}^{3} \mathrm{~g}^{-1}$ dans le cas où la décomposition se ferait à $973 \mathrm{~K}$.

Nous pouvons par conséquent conclure que la vapeur d'eau catalyse la décomposition du carbonate de magnésium, surtout à basse température, rendant le produit oxydé instable et provoquant des effondrements localisés au niveau microstructurel. L'effet de frittage de l'oxyde de magnésium à l'intérieur des grains, qui maintiennent leur volume global,

8. Harker et Tuttle (Harker et al., 1955, p. 212) avaient déjà observé que "if the pressure vessel was quenched before the pressure inside it was released, it was found that both lime and periclase could react to some extent with $\mathrm{CO}_{2}$ to form carbonates during the quench even though, when cold water was used, this took only about 15 seconds." 


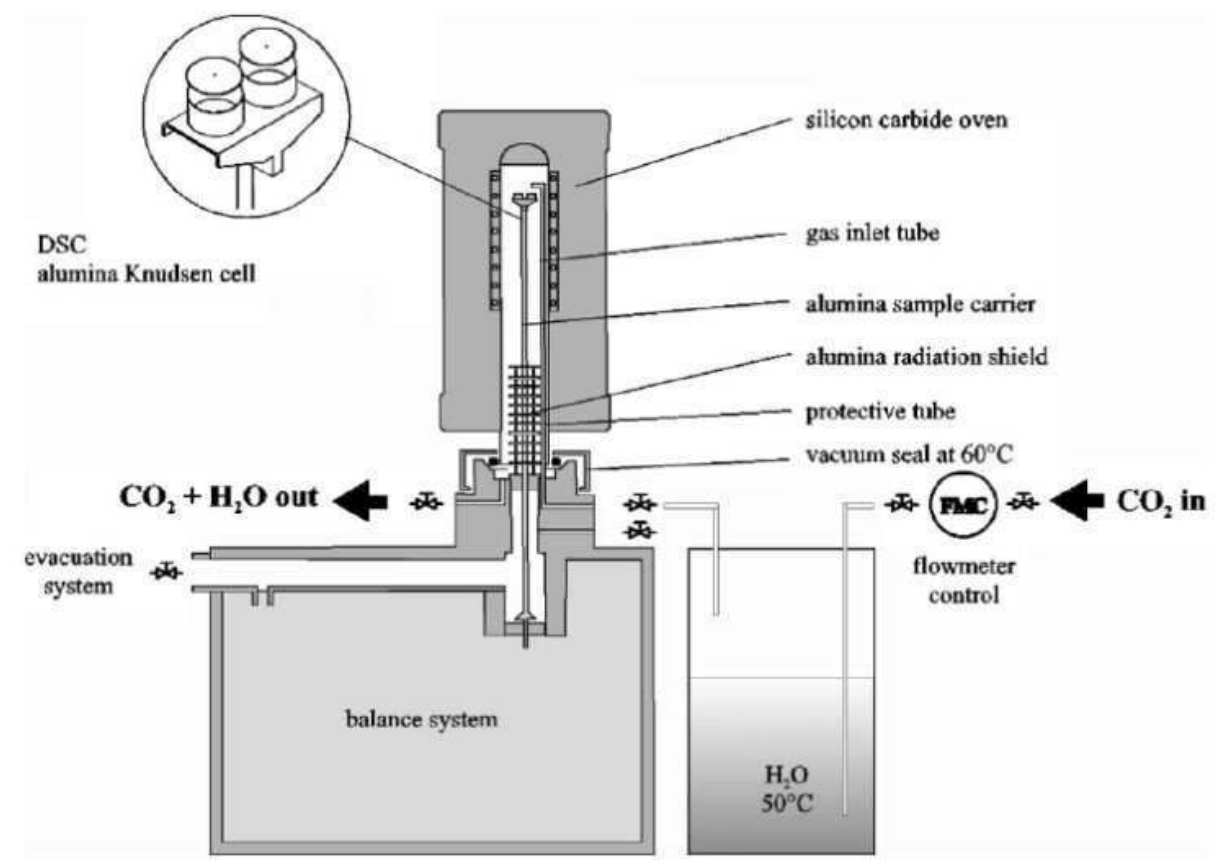

Figure 3 : Schéma de fonctionnement de l'appareil (TG - DSC) utilisé pour décomposer la poussière de dolomie en atmosphère de $\mathrm{CO}_{2}+\mathrm{H}_{2} \mathrm{O}$.

Figure 3: Device for changing dolomitic lime in $\mathrm{CO}_{2}+\mathrm{H}_{2} \mathrm{O}$

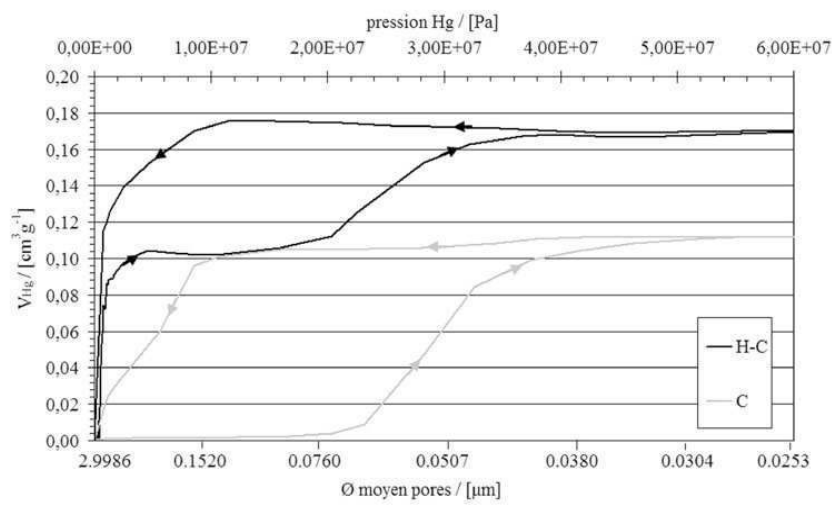

Figure 4 : Comparaison entre les tracés d'intrusion et d'extrusion du mercure des deux échantillons des produits solides obtenus par la décomposition partielle de la poussière de dolomie en atmosphère de $\mathrm{CO}_{2}(\mathrm{C})$ et de $\mathrm{CO}_{2}+\mathrm{H}_{2} \mathrm{O}(\mathrm{H}-\mathrm{C})$ à $913 \mathrm{~K}$.

Figure 4: Comparison between intrusion and extrusion of mercury.

augmente la porosité totale de l'échantillon et la dimension moyenne des pores.

En atmosphère constituée uniquement d'anhydride carbonique, les zones du produit oxydé sont par conséquent constituées de gros grains d'oxyde de calcium et de petits grains d'oxyde de magnésium non frittés. En atmosphère d'anhydride carbonique et de vapeur d'eau, aussi bien les zones d'oxyde de calcium que celles d'oxyde de magnésium, sont constituées par de gros grains, ce qui correspond à une plus grande porosité.

Les décompositions obtenues dans les fours à bois et donc en milieu riche en vapeur d'eau, produisent un oxyde de magnésium à grains plus gros et pores plus accessibles aux réactifs. Cette microstructure devrait avoir, par rapport à des phases liquides, une plus grande réactivité qui, dans le cas de l'extinction des oxydes, se traduit par une meilleure hydratation pour la formation de la chaux éteinte. La prochaine étape de la recherche consistera, effectivement, en l'évaluation de la réactivité des produits oxydés en présence de vapeur d'eau.

Dès lors, il semble possible de comprendre les indications fournies par Vallardi "... Certains ont pour usage de vaporiser de l'eau sur le combustible que l'on brûle dans le four, ou de jeter de l'eau dans le four au fur et à mesure que la cuisson avance : cet usage confirme l'influence favorable de la vapeur d'eau dans la décomposition du carbonate de chaux..." (Vallardi fin 1800, p. 280) ou par Misuraca selon lequel «... Sembra che una corrente di vapore acqueo, durante il periodo di demolizione per calcinazione del calcare, agevoli la decomposizione medesima. Era ritenuta perciò buona norma quella di mettere un recipiente con acqua sulla soglia della bocca da fuoco, durante l'operazione di cottura, perché il vapore acqueo 


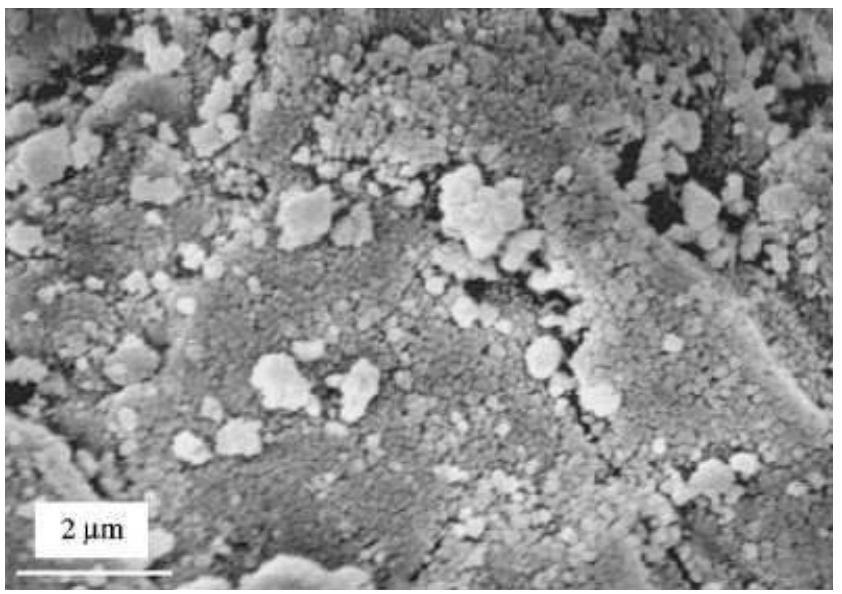

Figure 5: Microphotographie au SEM de poussière de dolomie partiellement décomposée en atmosphère de $\mathrm{CO}_{2}+\mathrm{H}_{2} \mathrm{O}$ à $913 \mathrm{~K}$ $(10.0 \mathrm{kx})$.

Figure 5: Scanning electron micrographs showing dolomitic dust partially break down into $\mathrm{CO}_{2}+\mathrm{H}_{2} \mathrm{O}$ in $913 \mathrm{~K}$.

potesse facilmente essere trasportato nella massa incandescente. ...» (Misuraca et al., 1900, 179) ${ }^{9}$.

\section{LA RECHERCHE SUR LE KAOLIN CUIT}

Pareillement à ce qui a été fait pour les chaux dolomitiques, le kaolin, une des principales composantes des mortiers réalisés à Gênes entre le Moyen Âge et l'époque contemporaine, est aujourd'hui, lui aussi, la cible d'attention de la part des chercheurs.

Contrairement cependant à ce que l'on a vu pour la recherche en laboratoire conduite sur les liants, qui a déjà atteint de remarquables niveaux d'approfondissement, les études sur le kaolin (commencées, à Gênes, à partir de la moitié des années quatre-vingt du siècle dernier) sont en cours d'approfondissement, et seulement dans les prochains mois il est prévu qu'elles atteignent les premiers résultats pour accroître les connaissances sur les thèmes traités.

L'étude vient de la nécessité de chercher à connaître plus profondément le système chaux/kaolin qui n'intéresse qu'une petite partie de la recherche scientifique la plus avancée, mais qui retient l'attention des chercheurs, lesquels ont déjà eu l'occasion de présenter les résultats atteints. Parmi

9. «...Il semblerait que, pendant la période de démolition pour la calcination du calcaire, la vapeur d'eau courrante favorise la décomposition elle-même. C'est ainsi qu'il était considéré de bonne règle le fait de placer un récipient rempli d'eau sur le bord de la bouche à feu, pendant l'opération de cuisson, afin que la vapeur d'eau puisse facilement être transportée dans la masse incandescente..." (traduction des auteurs). les études publiées sont comprises les recherches faites sur les sources orales et sur les sources matérielles par un des auteurs (Mannoni, 1988), les expérimentations réalisées par un groupe de chercheurs génois pour restituer la " recette " des mortiers au kaolin (Cucchiara et al., 1993), et plusieurs microanalyses semi quantitatives réalisées au SEM avec la méthode de la spectroscopie à dispersion d'énergie (EDS) sur les échantillons les plus significatifs du port de Gênes remontant à la fin du Moyen Âge (Giordani et al., 1999).

En plus de ces études, principalement conduites sur des échantillons de mortier, cette recherche se propose de vérifier, en analysant un ample nombre de cas, les modalités d'utilisation et les caractéristiques des produits obtenus avec l'emploi du kaolin dans la façon de construire génoise et ligure. Cette vérification pourra être développée sur la base des résultats d'une vaste série d'analyses en diffractométrie des rayons $\mathrm{X}$ conduites sur les échantillons de mortier prélevés à Gênes et dans toute la Ligurie, entre juillet 1985 et mai 2002.

La décision d'utiliser les résultats des analyses en diffractométrie comme instrument pour l'évaluation de l'utilisation du kaolin dans la façon de construire génoise et ligure dérive de la disponibilité d'une vaste banque de données (brièvement décrite par la suite), mais aussi du constat, déjà exposé dans les premiers travaux d'enquête réalisés, selon lequel l'une des méthodes les plus répandues dans l'analyse des mortiers, et utiles à l'identification de ce matériau particulier, est précisément l'analyse de la diffraction des rayons $\mathrm{X}$ qui, clairement, limite - mais n'infirme pas - les évaluations ici exposées. Si on ne développe des idées que sur les données des diffractométries, il est évident, en effet, que tout résultat pouvant être atteint ne pourra se référer qu'aux seules phases cristallines présentes dans les échantillons, et non aux phases microcristallines ou amorphes qui peuvent s'être formées au même moment et qui, comme on le sait, sont caractéristiques des mortiers et des liants hydrauliques (Cucchiara et al., 1993, 30).

Avant de procéder à l'exposé des idées susmentionnées, il est bon de s'arrêter encore brièvement sur le résultat des analyses qui seront discutées et, en particulier, il est utile de souligner certains aspects des évaluations quantitatives qui se rapportent à la seule kaolinite. En effet, étant activé thermiquement, le kaolin employé dans les mélanges est caractérisé par une phase minéralogique ayant un ordre à long rayon de très bas niveau. Ceci signifie que la kaolinite, quand elle est relevée par diffractométrie, ne peut apparaître qu'en traces et que celles-ci doivent être interprétées comme " impuretés ", à savoir les résidus d'une cuisson imparfaite de la matière première. Une activation complète du kaolin qui, étant donné la caractéristique de l'activité pyrotechno- 
logique conduite à l'époque préindustrielle (Giannichedda et al., 1996), ne pouvait être atteinte que dans de rares cas, aurait - en effet - rendu ce minéral complètement transparent à l'analyse XRD. Par conséquent, il en ressort qu'on ne peut attribuer aux possibles évaluations sur l'utilisation du kaolin qu'une erreur par défaut, ce qui peut donc porter à sous-estimer le phénomène examiné.

La banque de données actuellement à disposition est constituée de 155 analyses XRD conduites sur les mortiers. Le matériel collecté est constitué par les diffractogrammes interprétés des analyses réalisées sur tous les échantillons de mortier avec la méthode dite "des poussières".

Les données à disposition sur la nature et l'incidence des phases cristallines chaque fois identifiées ont été collectées dans une banque de données informatisée, réalisée en milieu MS Access, qui permet de faciliter les recherches par composé, par élément, par typologie de mélange (stuc, revêtement, mortier, etc.), par période de production, mais aussi par zone géographique de provenance. L'utilisation de l'outil informatique et l'organisation de la banque de données permettent ainsi le développement d'une série de réflexions sur les cas d'emploi, les finalités d'utilisation et sur les caractéristiques chimiques et minéralogiques des mortiers échantillonnés.

Si on examine les données insérées dans la banque de données, on peut facilement vérifier que des 155 mortiers analysés, seulement 12 , correspondant à $7,7 \%$ du total, ont mis en évidence la présence de kaolinite (Tableau 3).

De ce qu'on peut déduire en examinant plus en détail les résultats de ces analyses, il apparaît évident que la kaolinite a été utilisée tant dans les mortiers employés en milieux humides et/ou anaérobies, que dans des ouvres de finition dans lesquelles le niveau d'humidité et les difficultés de pénétration de l'anhydride carbonique résultent dans l'ensemble peu importants. À la lumière de ce large éventail de situations il est, en effet, possible de donner en exemple les mortiers du port de Gênes datant de la fin du Moyen Âge (échantillons 102, 103, 104, 105 et 106), et les échantillons de stuc et de revêtement prélevés dans différents lieux de la Ligurie.

Dans le premier cas la fonction de liant hydraulique propre du kaolin cuit semble constituer la principale motivation qui a conduit à l'adoption de ce matériau, tandis que dans les derniers cas reportés les motivations qui ont conduit à l'utilisation du kaolin sont encore objet de discussion de la part des chercheurs (il paraît que le kaolin a pu donner une certaine plasticité au mélange).

Si on entre plus en détail dans certaines de ces analyses, il est possible d'observer comment les échantillons du port de Gênes sont caractérisés par la présence de calcite, de kaolinite (toutes les deux en traces ou, tout au plus, en phases accessoires), de quartz (présent en quantité accessoire ou pré- dominante), de serpentine et/ou de feldspath (présent eux aussi en traces ou, tout au plus, comme phases accessoires), mais aussi de portlandite (échantillons 103, 105 et 106; phase prédominante ou accessoire), de dolomite (en traces ou accessoire) et ettringite (phase prédominante ou, tout au plus, accessoire). Si on tente d'interpréter ces données, il est possible de supposer de quelle manière la dolomie, la serpentine, le feldspath et une partie du quartz ${ }^{10}$ peuvent être rapportés au matériau réuni dans les mélanges ayant une fonction de dégraissage, d'anti-retrait, etc.; tandis que la présence de portlandite doit être attribuée à la phase liante.

La présence de portlandite, relevée aussi en grande quantité en association à une présence limitée de carbonates de néo-formation, semble être la conséquence du milieu d'utilisation dans lequel de tels mélanges ont été employés, qui résulte d'une faible présence d'anhydride carbonique (milieu anaérobie). En revanche, l'ettringite peut être envisagée comme phase de néo-formation produite par les réactions qui peuvent se développer entre l'hydroxyde de calcium et la metakaolinite quand il y a du soufre dans les systèmes, dans ce cas amené par l'alun, présent comme impureté dans le kaolin lui-même. Les gisements de ce matériau étaient, en effet, contigus à ceux du kaolin probablement utilisé à Gênes et, en outre, les deux éléments se trouvent souvent " naturellement " mélangés ensemble (Cucchiara et al., 1993, 28; Giordani et al., 1999) ${ }^{11}$. Dans ce cas, il est intéressant d'observer comment le sulfate ci-dessus indiqué se présente dans presque tous les cas en quantités très élevées qui, comparées à celles des sulfates contenus naturellement dans les stucs ou ajoutés ici par un processus de dégradation par sulfatation, se révèlent excessives.

Si on focalise l'attention sur les stucs au kaolin, il est possible d'observer combien les analyses de l'échantillon n. 87, prélevé dans un des édifices de la ville de Gênes, rendent compte d'un mortier constitué par peu de phases minérales : gypse (présent comme phase prédominante), carbonate de calcium (relevé seulement en traces et, par ailleurs, sous forme d'aragonite et non de calcite), kaolinite et quartz (tous les deux relevés en traces). Dans ce cas le gypse peut être identifié comme principale phase liante utilisée dans le mélange, tandis que l'aragonite peut être considérée comme une nouvelle formation et attribuée à la réaction de carbonatation de la chaux utilisée comme phase liante

10. Une partie du quartz peut être, en effet, attribuée au squelette du kaolin, tandis qu'une autre partie l'est aux sables. À l'état actuel des connaissances il n'est, cependant, pas possible de faire la distinction entre les deux sources (Cucchiara et al., 1993, p. 26).

11. À ce propos on peut donner l'exemple de l'analyse chimique du Kaolin de la Tolfa, ainsi composé $: \mathrm{SiO}_{2}=55,82 \% ; \mathrm{Al}_{2} \mathrm{O}_{3}=18,31 \%$; $\mathrm{Fe}_{2} \mathrm{O}_{3}=1,25 \% ; \mathrm{CaO}=1,14 \% ; \mathrm{MgO}=0,38 \% ; \mathrm{SO}_{3}=9,89 \%$. 


\begin{tabular}{|c|l|c|c|c|c|}
\hline$N^{\circ}$ & \multicolumn{1}{|c|}{ Type } & Commune & Province & Utilisation & Note \\
\hline 27 & Stuc & Gorra & Imperia & Stuc & $\begin{array}{l}\text { Le stuc présentait problèmes de } \\
\text { dégradation }\end{array}$ \\
\hline 41 & Gaslini & Gênes & Gênes & - & - \\
\hline 53 & Palazzo Reale & Gênes & Gênes & - & - \\
\hline 55 & Spezia 2 & La Spezia & $\begin{array}{c}\text { La } \\
\text { Spezia }\end{array}$ & - & - \\
\hline 87 & Stuc & Gênes & Gênes & Stuc & Utilisé en intérieurs \\
\hline 92 & Revêtement & - & - & Revêtement & - \\
\hline 102 & Port de Gênes & Gênes & Gênes & Mortier & - \\
\hline 103 & Port de Gênes & Gênes & Gênes & Mortier & - \\
\hline 104 & Port de Gênes & Gênes & Gênes & Mortier & - \\
\hline 105 & Port de Gênes & Gênes & Gênes & Mortier & - \\
\hline 106 & Port de Gênes & Gênes & Gênes & Mortier & - \\
\hline 186 & Pont Greazzola & Gênes & Gênes & Mortier & \\
\hline
\end{tabular}

\begin{tabular}{|c|c|c|c|c|c|c|c|c|}
\hline \multirow{2}{*}{$\mathrm{N}^{\circ}$} & Hydroxydes & \multicolumn{9}{|c|}{ Carbonates } & \multicolumn{3}{c|}{ Sulfates } \\
\cline { 2 - 9 } & Portlandite & Calcite & Aragonite & Magnésite & Dolomie & Gypse & Epsomite & Ettringite \\
\hline 27 & - & + & - & + & - & + & +++ & - \\
\hline 41 & - & ++ & - & - & - & - & - & - \\
\hline 53 & - & +++ & - & ++ & - & + & - & - \\
\hline 55 & - & +++ & - & - & - & - & - & - \\
\hline 87 & - & - & + & - & - & +++ & - & - \\
\hline 92 & - & +++ & ++ & - & - & - & - & - \\
\hline 102 & - & ++ & - & - & + & - & - & +++ \\
\hline 103 & ++ & ++ & - & - & ++ & - & - & ++ \\
\hline 104 & - & + & - & - & + & - & - & +++ \\
\hline 105 & +++ & ++ & - & - & + & - & - & +++ \\
\hline 106 & +++ & ++ & - & - & ++ & - & & - \\
\hline 186 & - & ++ & - & - & - & - & & + \\
\hline
\end{tabular}

\begin{tabular}{|c|c|c|c|c|c|c|}
\hline \multirow{2}{*}{$\mathrm{N}^{\circ}$} & \multicolumn{2}{|c|}{ Silicates hydratés } & \multicolumn{5}{c|}{ Silico-aluminates hydratés } \\
\cline { 2 - 7 } & Antigorite & Smectite & Illite & Muscovite & Chlorite & Clinochrysotile \\
\hline 27 & - & - & + & - & - & - \\
\hline 41 & - & $?$ & $?$ & - & $?$ & - \\
\hline 53 & ++ & - & - & - & - & - \\
\hline 55 & - & - & & + & - & - \\
\hline 87 & - & - & - & - & - & - \\
\hline 92 & - & - & - & - & - & + \\
\hline 102 & - & - & - & - & - & - \\
\hline 103 & - & - & - & - & - & - \\
\hline 104 & - & - & - & - & - & - \\
\hline 105 & - & - & - & - & - & - \\
\hline 106 & - & - & - & - & - & - \\
\hline 186 & - & - & + & - & - & - \\
\hline
\end{tabular}

\begin{tabular}{|c|c|c|c|c|c|c|}
\hline \multirow{2}{*}{$\mathrm{N}^{\circ}$} & \multicolumn{3}{|c|}{ Silico-aluminates } & \multicolumn{3}{c|}{ Autre } \\
\cline { 2 - 7 } & Kaolinite & Microcline & Albite & Serpentin & Feldspath & Quartz \\
\hline 27 & + & - & - & - & - & ++ \\
\hline 41 & $?$ & - & - & - & ++ & +++ \\
\hline 53 & + & - & $?$ & - & - & +++ \\
\hline 55 & + & - & - & - & - & +++ \\
\hline 87 & + & - & - & - & - & + \\
\hline 92 & + & - & - & - & - & +++ \\
\hline 102 & + & - & - & - & + & +++ \\
\hline 103 & ++ & - & - & + & ++ & +++ \\
\hline 104 & + & - & - & + & - & ++ \\
\hline 105 & + & - & - & + & - & ++ \\
\hline 106 & + & - & - & - & ++ & ++ \\
\hline 186 & + & ++ & - & - & ++ & +++ \\
\hline
\end{tabular}

Tableau 3 : Liste des échantillons de mortier contenant de la kaolinite. Description et énumération des phases minéralogiques identifiées en diffractométrie, accompagnées de la donnée quantitative $(+++=$ prédominant $;++=$ accessoire $;+=$ en traces $)$.

Tableau 3: Samples of mortars with kaolinite. 
- quantitativement - secondaire. Selon certaines études thermodynamiques, la présence de divers habitus cristallins du carbonate de calcium peut fournir des indications sur la vitesse de développement des réactions de prise et de solidification du mélange qui, dans ce cas, devrait avoir été un peu lente (Giordani et al., 1987; Moropoulou et al., 1993, 419) mais complète, vu l'absence d'hydroxydes présents dans les mortiers du port. La présence de kaolinite et le quartz (totalement ou en partie) sont, enfin, à attribuer à l'utilisation du kaolin dans le mélange (sans, d'ailleurs, une motivation claire).

Y compris dans le stuc provenant du village de Gorra qui, cependant, présentait quelques problèmes de dégradation, on observe la présence d'une quantité limitée de phases carbonatées (dans ce cas calcite et magnésite, toutes les deux relevées en traces) et une quantité élevée de sulfates, divisés de manière asymétrique entre gypse et epsomite, cette dernière étant la phase prédominante de tout l'échantillon (le gypse n'est présent qu'en traces). La kaolinite, comme toujours, est présente en traces, tandis que l'on a relevé une quantité plus élevée de quartz (phase accessoire) par rapport au cas précédemment illustré, et une quantité limitée d'illite. Même dans ce cas, on peut observer comment une partie des sulfates provient de l'utilisation du gypse dans la phase liante du mélange, tandis que la calcite provient de la réaction de carbonatation de la chaux (même dans ce cas il s'agirait de la phase liante secondaire). En revanche, l'epsomite est le composé typique de la dégradation par sulfatation des mortiers utilisant un liant magnésien qui, on le sait, a été très utilisé y compris dans ces zones de la Ligurie. Lillite et la magnésite peuvent être, enfin, prises comme phases minérales de l'agrégat, qui devait être partiellement quartzeux. Y compris dans ce cas, l'adjonction de kaolin (auquel il faut attribuer une partie de la silice du quartz) résulte clairement.

Si on porte l'attention sur les autres mélanges analysés, il ressort avec évidence que le revêtement est composé d'une grande quantité de phases carbonées (calcite en quantité prédominante et aragonite en quantité accessoire), d'une importante quantité de quartz (phase prédominante), de kaolinite et de clinochrysotile (les deux en traces). L'interprétation de ces données fait penser à un mélange réalisé avec de la chaux comme phase liante, par un agrégat partiellement quartzeux, mais aussi par du kaolin cuit utilisé, comme vu plus haut, sans une fonction claire. La présence d'aragonite peut, comme dans le cas susmentionné, fournir des indications sur les modalités de développement des réactions de prise et de solidification.

En conclusion, comme déjà anticipé, il ressort de l'étude des mortiers au kaolin échantillonnés au cours de ces années de recherche que l'emploi de ce matériau a été très ample: la période d'utilisation va de la moitié du $\mathrm{Xv}^{\mathrm{e}}$ siècle au début du $\mathrm{xx}^{\mathrm{e}}$ siècle (Mannoni, 1988), tandis que les modalités d'utilisation comprennent tant les mortiers réalisés pour être employés en milieu humide, que les stucs et les induits les plus « simples " carbonatés à l'air.

Comme on peut voir, quand elle est présente, la kaolinite est presque toujours observée en traces et elle est rarement remarquable en quantités accessoires. Aussi dans les stucs l'utilisation du kaolin paraît, donc, liée à la formation de liants hydrauliques; c'est pour ça qu'on peut supposer que les deux revêtements échantillonnés (dont on n'a pas d'exemples ultérieurs) furent employés en maçonneries sujettes à phénomènes d'humidité (par exemple en présence d'une forte humidité de remontée, ou sujets aux averses régulières), lesquels exigeaient, donc, un liant au moins faiblement hydraulique. La documentation écrite de l'époque moderne, étudiée par les chercheurs engagés dans la reconstruction de la « recette " de ces mortiers particuliers, indique l'existence de revêtements hydrauliques avec kaolin, réalisés dans le but d'opposer une meilleure résistance à l'eau (Cucchiara et al., 1993).

De ce qu'on peut déduire en examinant les autres phases micro cristallines présentes dans les échantillons analysés, est que la présence de l'hydroxyde de calcium, et les divers habitus cristallins du carbonate de calcium, peuvent fournir des indications sur le milieu dans lequel se sont réalisées la réaction de prise et la solidification des mortiers.

Les sulfates résultent hydrates seulement dans les mortiers qui ont subi une prise et une solidification dans l'eau. En outre, quand ces composés sont relevés, ils se présentent en quantités très élevées qui, comparées à celles des sulfates normalement présents dans les autres mélanges, sont, elles aussi, excessives. Ceci pourrait, peut-être, découler de l'usage, non écrit, d'ajouter une certaine quantité d'alun aux mélanges kaoliniques, probablement dans le but d'améliorer les propriétés des mortiers grâce aux processus de réaction qui sont aujourd'hui encore objet d'étude de la part de plusieurs chercheurs (voir, entre autres, Cucchiara et al., 1993 et Böke, 2003).

De plus, il est intéressant d'observer l'absence de composés comme la gehlenite et la stratlingite, qui sont souvent présents dans de nombreuses recherches en laboratoire dans lesquelles on explore les processus de réaction entre le kaolin cuit et la chaux. La formation de ces phases, dans ces cas, serait en effet à attribuer à une procédure incorrecte de préparation des mélanges, qui sont réalisés en hydratant un mélange de kaolin et de carbonate de calcium, cuits ensemble. Mélange qui, comme on le sait, produit pendant la cuisson les composés mentionnés ci-dessous.

ArCheoSciences, revue d'archéométrie, 30, 2006, p. 67-79 
L'unique cas d'identification des pics de diffraction de la gehlenite parmi les analyses archivées, est celui des échantillons provenant d'un four localisé dans les environs immédiats de la ville de Gênes (cas non reporté dans le tableau en annexe) qui, pour cela, peut peut-être représenter comme l'unique cas actuellement connu de four pour la cuisson du kaolin. La formation de la gehlenite, dans ce cas, serait donnée par la réaction à l'état solide entre la calcite du mortier utilisé pour construire le four et la kaolinite ici placée pour l'activation, selon une réaction largement connue en littérature (Traoré, 2003).

\section{Conclusion}

Dans l'ensemble on peut, donc, affirmer que les études menées aux laboratoires de la Faculté d'Ingénierie des matériaux permettent d'aller plus loin que les résultats attendus, ainsi que comprendre, non seulement les caractéristiques chimiques-physiques, les modalités d'utilisation et la technologie de production des mortiers réalisées à Gênes à partir de la fin du Moyen Âge, mais aussi la culture matérielle et le savoir faire des anciens maîtres constructeurs.

En effet il serait très difficile d'acquérir ces connaissances de manière différente, puisqu'elles appartiennent au savoir immatériel, caractéristique de tous les arts; le moyen de placer des cuves à l'embouchure des fours à chaux dans le but d'améliorer la qualité du produit, ou les variations des « recettes " des mortiers au kaolin, donnés par les diverses conditions d'utilisation (par exemple l'adjonction ou moins d'alun aux mortiers qui devaient être employés dans les ouvrages portuaires) représentent seulement un exemple de ces connaissances.

Ça veut dire qu'à partir au moins du $\mathrm{XI}^{\mathrm{e}}$ siècle, tous les choix réalisés au niveau de filières de production, qui sont liées à la réalisation des mortiers pour la construction (du choix du type de roche utilisable pour la production de la chaux, aux modalités de cuisson de celle-ci) avaient lieu sur la base de connaissances empiriques bien définies, et elles étaient probablement le fruit d'un processus continu d'amélioration des savoirs faire acquis pendant le temps.

L'introduction de l'utilisation du kaolin comme additif hydraulique dans les mortiers de chaux magnésienne, remontant au Xv $\mathrm{Xv}^{\mathrm{e}}$ siècle, s'est basée, au contraire, sur l'importation d'expériences de différentes cultures de construction (probablement d'origine byzantine; Mannoni, 1988), mais le développement de cette technologie a permis aux constructeurs génois la réalisation d'ouvrages qui sont sans égal encore aujourd'hui pour leur résistance élevée aux agents chimiques et biologiques.
La poursuite des études aux laboratoires d'ingénierie des matériaux, au contact avec les chercheurs qui opèrent dans le domaine archéologique, permettra peut-être, dans un futur prochain d'obtenir des résultats ultérieurs, à l'heure actuelle encore inespérés, comme c'est passé dans ces dernières années. Le nombre limité des chercheurs intéressés à ces thèmes est tel que, aujourd'hui plus que jamais, est nécessaire une comparaison continue et un échange d'expériences entre les experts de divers pays et de différentes disciplines.

\section{Bibliographie}

Allasinaz, A., Gelati, R., Gnaccolini, M., Martinis, B., Orombelli, G., Pasquarè, G. et Rossi, 1971. Carta geologica d'Italia. f. 82, Nuova Tecnica grafica, Roma.

Bamford, C. H. et Tipper, C. F. H., 1980. Chemical Kinetics, Amsterdam, C. H. Bamford and C. F. H. Tipper.

BöKE, H., 2003. Ettringite formation in historic bath brick-lime plaster, Cement and Concrete Research, 33, p. 1457-1464.

Commissione Europea e Centro Comune di Ricerca, 1995. Fornaci da calce in provincia di Varese. Storia, conservazione e recupero. Atti del convegno di studi, Arte Stampa, Daverio.

Cucchiara, A., Mannoni, T., Montagni, C., Negretti, L., Predieri, G., Ricci, R. et Sfrecola, S., 1993. I calcestruzzi "alla porcellana" in Liguria, in G. Biscontin, Scienza e Beni Culturali, Bressanone, IX, Padovan Editore, 21-30.

GHERSI, I., 1903. Imitazioni e succedanei nei grandi e piccoli prodotti industriali, Milano, Hoepli.

Giannichedda, E. et Mannoni, T., 1996. Archeologia della produzione, Torino, Einaudi.

Giordani, M. et Beruto, D., 1987. Effect of vaporization rate on calcium carbonate nucleation from calcium hydrogen carbonate aqueous solution, Journal of Cystal Growth, 84, p. 679-682.

Giordani, M. et Mannoni, T., 1999. La tecnica degli antichi maestri muratori : identificazione di uno stadio del processo lavorativo attraverso l'analisi chimica e mineralogica di malte idrauliche storiche, in AA.VV., Atti del II convegno : Materiali e Tecniche per il restauro, Cassino, Idea Stampa Editore, p. 9199.

Harker, R. I. et Tuttle, O. F., 1955. Studies in the system $\mathrm{CaO}-\mathrm{MgO}-\mathrm{CO}_{2}$. Part 1 : the thermal dissociation of calcite, dolomite and magnesite, American Journal of Science, 253, p. 209-224.

Lenormand, L., Payen, L. et Molard, J., 1839. Nuovo Dizionario Universale Tecnologico o di arti e mestieri e della economia industriale e commerciante. XXIII, G. Antonelli, Venezia.

MaEde, R., 1909. Richard Maede Cement age, Revue de Matériaux de Construction. 
ManNoni, T., 1988. Ricerche sulle malte genovesi alla "porcellana", in G. Bisconti, Le Scienze, le istituzioni, gli operatori alla soglia degli anni '90, Bressanone, Padovan Editore, p. 137a-142a.

Manzano, E., Bueno, A. G., Gonzalez-Casado et A., Monsalud DEL Olmo, 2000. Mortars, pigments and binding media of wall paintings in the 'Carrera del Darro' in Granada, Spain, Journal of Cultural Heritage, 1: 19-28.

Margrave, J., 1960. The Characterization of High Temperature Vapors, Wiley, New York.

Misuraca, G., Boldi, M. A. et Aurelio, M., 1900. L'arte moderna di fabbricare : trattato pratico ad uso degli ingegneri, costruttori, capimastri e studenti, Vallardi, Milano.

Mohazzabi, P. et SEarcy, A. W., 1974. Journal of the Chemical Society, $70: 2145$.

Moropoulou, A., Biscontin, G., Bisbikou, K., BaKolas, A., Theoulakis, P., Theodoraki, A. et Tsiourva, T., 1993. Physico-chemical study of adhesion mechanism among binding material and brick fragments in "cocciopesto", in AA.VV., Atti del Convegno Scienza e Beni Culturali, IX, Bressanone, Padovan Editore, p. 415-429.

Newton, R. G. et Sharp, J. H., 1987. An investigation of the chemical constituents of some renaissance plasters, Studies in Conservation, p. 163-175.
Pareto, R. et Sacheri, G., 1885. Enciclopedia delle Arti e delle Industrie, Unione tipografico editrice, Torino.

Società Geologica Italiana 1994. Guide Geologiche Regionali : Alpi Liguri, BeMa editrice, Milano.

STERN, K. H., 1969. High Temperature Properties and Decomposition of Inorganic Salts, Washington, Government Printing Office.

Tamagno, E., 1987. Fornaci : terre e pietre per l'ars aedificandi, Torino, Umberto Allemandi.

TraORÉ, F., 2003. Gehlenite e anortite crystallization from kaolinite and calcite mix, Ceramics International, 29, p. 377-383.

Vallardi, fin 1800. Dizionario Industriale di Arti e Mestieri.

VeCCHiattini, R., 1998. Unità produttive perfettamente organizzate : le calcinare di Sestri ponente - Genova, Archeologia dell'Architettura, 3, p. 141-152.

ViCAT L. J., 1856. Traité pratique et théorique de la composition des mortiers, ciments et gangues à pouzzolanes et de leur emploi dans toutes sortes de travaux, suivi des moyens d'en apprécier la durée dans les constructions à la mer, Grenoble, Imprimerie Maisonville. 